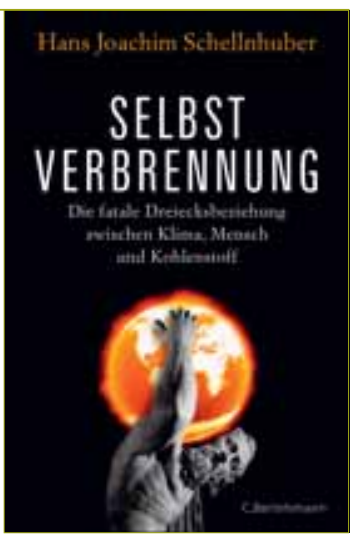

Schellnhuber, H. J. 2015. Selbstverbrennung. Die fatale Dreiecksbeziehung zwischen Klima, Mensch und Kohlenstoff. München: C. Bertelsmann. 784 S., 29,99 EUR, ISBN 978-3-570-10262-6

\title{
Die schmerzhafte Einsicht in die selbstzerstörerischen Folgen der Erderwärmung
}

\author{
Ein autobiografischer, historischer, politischer und \\ kultureller Blick auf die vom Menschen jüngst angelegte \\ Klimageschichte zeigt die Ausnahmesituation, in die \\ der Eingriff des Menschen in den Kohlenstoffkreislauf \\ das Leben auf der Erde absehbar gebracht hat.
}

Julia Hertin, Hans-Jochen Luhmann

\section{Intention}

Das rund 800-seitige Werk Selbstverbrennung des deutschen Klimaforschers Hans Joachim Schellnhuber irritiert - mit Titel, Gliederung und Inhalten. „Selbstverbrennung“ statt etwa „gefährlicher menschgemachter Klimawandel“ - das bezieht sich wörtlich auf die Einschränkung von Lebensbedingungen schon physiologisch, durch Hitzestress. Aber Schellnhuber greift das Motiv der „Verbrennung“ auch metaphorisch auf, zum Beispiel in der Diagnose, „die naiven Verheißungen der Moderne“ stünden „in Flammen“.

Schellnhuber geht es im Kern um eine neue Ansprache der Öffentlichkeit vonseiten der Wissenschaft - und das nicht programmatisch, sondern im Modus des Selber-Tuns. Wissenschaft kommuniziert primär im Selbstgespräch, die Öffentlichkeit anzusprechen bedarf einer „Übersetzungsleistung“ - diesen Anspruch erfüllt er radikal, ohne Scheu vor innerwissenschaftlichen Tabubrüchen. So ersetzt er emotional neutrale beziehungsweise „wertfreie“ Begriffe der etablierten Wissenschaft, von der das Buch handelt, durch packende, ansprechende Bilder. Zudem gibt er Einblicke in sein Erleben als verletzliche Person, bei der Herausbildung seiner Einsicht in die heutige geschichtliche Stunde.

„Zeugnis ablegen“ nennt er das Genre seines Buchs - von dem man sich schwer vorstellen kann, dass danach noch ein weiteres folgt. Er wirft den eh ideologischen, also methodologisch unbegründeten $\mathrm{Ge}$ gensatz von Objektivität und Subjektivität auch explizit über Bord, bringt folglich sich als Person mit seiner Geschichte, seinen persönlichen zeitgenössischen Beobachtungen ein. Schließlich verstößt er gegen die eherne Wissenschaftsregel, dass „ein Experte (...) größtmöglichen Abstand von abgestuft: Manches gehe nur knapp unter die Haut, anderes treffe einen im Fleisch, Zentrales aber berühre einen im Mark.

Das inhaltliche Spektrum des Buchs reicht von der Historie der Klimaforschung - das Beste, was im wissenschaftlichen

\section{Unterhaltsam, sprachgewaltig, bisweilen sogar poetisch ...}

den moralischen Dimensionen seiner Thematik zu wahren hat". Das betreffe nur den Normalbetrieb. Angesichts der Größe der kollektiv anstehenden Herausforderung gelten die Sitten, die im Normalbetrieb angemessen sind, nicht mehr.

\section{Aufbau und Inhalt}

Sein Werk umfasst drei Hauptkapitel, betitelt mit Die Haut, Das Fleisch, Das Mark. Mit dieser Gliederung aus Begriffen der Leiblichkeit drückt Schellnhuber seine Überzeugung aus, dass die Verflechtung „wissenschaftlicher Einsichten, persönlicher Erinnerungen und politisch-moralischer Wertungen“ einen nichtlinearen Aufbau erfordere. Seine Unorthodoxie geht so weit, dass er sich sogar als Autor dieser Gliederung zugunsten der „inneren Logik“ zurückzieht, die ihm „der Gegenstand selbst aufgezwungen“ habe („nur [...] Stenotypist im Dienste des eigentlichen Autors"). Die mit Leiblichkeit verknüpften Kapitelüberschriften sollen vermutlich assoziieren lassen, dass alles berührt - jedoch
Schrifttum zu diesem Thema zu finden ist; das verdiente eine getrennte Publikation als Monografie - bis zum Insiderbericht aus den internationalen Klimaverhandlungen, von möglichen technologischen Antworten auf die drohende Klimakrise bis zur Auseinandersetzung mit den Leugner(inne)n des Klimawandels. Unterhaltsam, sprachgewaltig, bisweilen sogar poetisch gibt er Forschungsstände verschiedenster Disziplinen wieder, entfaltet große sozioökonomische Zusammenhänge und veranschaulicht die Persönlichkeit wichtiger

Kontakt: Dr. Julia Hertin | Sachverständigenrat für Umweltfragen (SRU) | Berlin | Deutschland | E-Mail: julia.hertin@umweltrat.de

Dr. Hans-Jochen Luhmann | Wuppertal Institut für Klima, Umwelt, Energie GmbH Wuppertal | Deutschland | E-Mail: jochen.luhmann@wupperinst.org

(C) 2016 J. Hertin, H.J. Luhmann; licensee oekom verlag. This is an article distributed under the terms of the Creative Commons Attribution License (http://creativecommons.org/licenses/by/3.0), which permits unrestricted use, distribution, and reproduction in any medium, provided the original work is properly cited. 
Akteure in Klimawissenschaft und -politik. Die Hoffnung, großskalige Klimainterventionen (Geoengineering) könnten die Erderwärmung abbremsen, hält er für ein Beispiel derselben menschlichen Hybris, die den Klimawandel herbeigeführt hat.

\section{Verhältnis Wissenschaft - Politik}

Entscheidend für Schellnhuber ist, dass der selbstzerstörerische Kurs der Menschheit von zentralen Akteuren geleugnet und politisch faktisch verdrängt wird. Dass dies gelingt, führt er auch auf die fehlende gesellschaftliche Durchschlagskraft der Wissenschaft sowie ihre defiziente Kommunikation zurück. Seine persönliche Konsequenz, die der 66-Jährige in diesem Buch verkündet: Er wolle „nunmehr endgültig Partei (...) ergreifen - gegen eine gesellschaftliche Betriebsweise, welche die natürlichen Lebensgrundlagen unweigerlich zerstören wird“ (S. 6). Dieses Bekenntnis wirft die Frage nach einem produktiven Verhältnis zwischen Wissenschaft und Politik auf. Einerseits entspricht der Duktus des Buchs in weiten Teilen dem sogenannten linearen Modell der wissenschaftlichen Politikberatung, des speaking truth to power: Wissenschaftler(innen), deren Legitimation sich aus der Kenntnis des Stands der Wissenschaft speist, informieren die Politik, die auf dieser Basis besser informierte Entscheidungen zu treffen vermag. Gleichzeitig grenzt sich Schellnhuber jedoch gegen die für dieses Modell typische Trennung von Wissenschaft und Politik ab: Da heute nichts mehr normal auf diesem Planeten sei, wird der Wissenschaftler aus dem „Zusammentreffen von Einsicht und Moral“ zum „Gewissenschaftler“, der sich für das ethisch Richtige einsetzt. Dass das Verhältnis zwischen Wissenschaft und Politik faktisch ein deutlich komplexeres ist als mit dem linearen Modell theoretisch debattiert, müssen sie sorgsam mit der Frage umgehen, wo Ergebnisse und Empfehlungen subjektiv geprägt werden.

\section{Widerstand gegen den fossilen Pfad}

Das Buch fordert dazu auf, sich der historischen Entscheidungssituation bewusst zu werden und die „Komplizenschaft“ im „klimazerstörerischen fossilen Betriebssystem“ aufzukündigen. Gemeint ist da-

\section{Schellnhuber fordert einen verstärkten Widerstand gegen den dominierenden fossilen Wirtschaftspfad.}

wird in diesem Buch besonders deutlich. So beschreibt der Autor, wie Wissenschaftler(innen) die Klimapolitik auch jenseits harter wissenschaftlicher Fakten prägen. Konzepte wie die Zwei-Grad-Leitplanke und der Budgetansatz, ein Prinzip zur Aufteilung der noch akzeptablen $\mathrm{CO}_{2}$-Emissionen auf einzelne Staaten, haben eine wissenschaftliche Grundlage, sind allerdings gleichzeitig normativ durchdrungen, das heißt transwissenschaftlich. Die Grenze zwischen Wissenschaft und Politik ist also keine statische Demarkationslinie, sondern muss in der breiten Grauzone transwissenschaftlicher Fragestellungen stets neu verhandelt werden. Da Wissenschaftler(innen) nicht neutral bleiben können, mit nicht (nur) das individuelle ökologische Verhalten vom Energiesparen bis zum Fahrradfahren. Vielmehr fordert Schellnhuber auch einen persönlich getragenen, politisch verstärkten Widerstand gegen den noch dominierenden fossilen Wirtschaftspfad ein. Inzwischen ist auch für ihn die politischste aller Fragen die nach dem persönlichen Beitrag - kein Problem der Moderne sei so mit den Gewohnheiten und Entscheidungen des/der Einzelnen verknüpft wie die Klimakrise.

Weshalb aber Schellnhuber, der in Klimafragen Systemiker par excellence ist, in Sachen Politik, vor allem Wissenschaftspolitik, den Gegen-Systemiker gibt, bleibt den Leser(inne)n ein Rätsel. 